\title{
Radiation induced pemphigoid disease
}

\author{
Jihyun Kim, MD, Taewon Jung, MD, Kyongjin Kim, MD, PhD, KyungA Kim, MD, MS, Sangki Hong, MD, \\ Sukyung Baek, MD, Jeongbum Moon, MD, MS
}

Department of Obstetrics and Gynecology, Presbyterian Medical Center, Jeonju, Korea

Among the possible complications of radiation therapy, acute and chronic side effects on the skin can be induced by percutaneous radiotherapy in the target site. Common skin lesions include radiation dermatitis, which can be treated by topical application of dressing and ointment. Pemphigoid disease, which displays similar clinical features as other skin diseases such as recurrent cancer and herpes zoster, rarely occurs in the site of radiotherapy; therefore, care must be taken during diagnosis for a timely treatment. The present report is a case of pemphigoid disease that had developed in a patient with endometrioid/clear cell carcinoma after radiation therapy, and the time between onset and radiotherapy was more than 6 months.

Keywords: Pemphigoid; Endometrial cancer; Radiation therapy

\section{Introduction}

Pemphigoid is subepidermal autoimmune disease associated with internal malignancies including endometrial cancer and it has various clinical manifestation. Erosions and erythema with multiple blisters develop all over the body. After administration of radiotherapy, this rare complication can occur in the irradiated area. Also there are several chemotherapeutic agents have many side effects including skin toxicity, and it significantly worsens the quality of life of patients. Long term Radiation along with chemotherapy can easily harm on skin barrier, and its disruption often cause autoimmune blistering disease.

We report a case of radio-induced bullous pemphigoid in elderly woman, who was treated for endometrial cancer and suffered pemphigoid after the radiation therapy, treated with steroid therapy.

\section{Case report}

A 66-year-old female patient who was diagnosed with endometrioid/clear cell carcinoma had progression to stage $\mathrm{lb}$ after radical hysterectomy. She received adjuvant chemotherapy with paclitaxel/carboplatin. There was no response to first-line chemotherapy, and a possible brain metastasis was suspected on computed tomography. Thus, chemotherapy was terminated, and she underwent whole-pelvis and wholebrain radiotherapy for 3 months.

Eight months later, she revisited the hospital for pain from bullae and erosion in the right thigh. A topical corticosteroid cream and an antihistamine were prescribed for allergic dermatitis or radiation dermatitis. However, the bullae continued to spread. Therefore, valaciclovir and acyclovir creams were administered for 7 days, after which she was admitted, as the bullae over $0.5 \mathrm{~cm}$ in size further expanded to $5 \mathrm{~cm}$ and the erosion worsened (Fig. 1). During this new hospitalization, the lesion site was cleansed daily with saline, a hydrogel dressing was applied, and mupirocin ointment and triaxone injection were administered. After 1 month of treatment, the inflamed area healed, but the spread and bursting of the bullae increased. Ten days later, prednisolone $10 \mathrm{mg}$ QD was

Received: 2020.03.19. Revised: 2020.08.05. Accepted: 2020.08.23. Corresponding author: Kyongjin Kim, MD, PhD

Department of Obstetrics and Gynecology, Presbyterian Medical Center, 365 Seowon-ro, Wansan-gu, Jeonju 54987, Korea

E-mail: kyongjink@gmail.com

https://orcid.org/0000-0002-9085-6958

Articles published in Obstet Gynecol Sci are open-access, distributed under the terms of the Creative Commons Attribution Non-Commercial License (http://creativecommons. org/licenses/by-nc/3.0/) which permits unrestricted non-commercial use, distribution, and reproduction in any medium, provided the original work is properly cited.

Copyright $\odot 2020$ Korean Society of Obstetrics and Gynecology 


\section{Obstetrics \& Gynecology Science}

Jihyun Kim, et al. Radiation induced pemphigoid disease

administered along with the above antibiotics and mupirocin ointment (Fig. 2). Furthermore, no bacterial isolates were found; therefore, we maintained the injectable antibiotic regimen. After 10 days, the bullae spread ceased, and for the next 7 days, the dosage was reduced to $5 \mathrm{mg}$. The wounds healed after the administration of a diuretic for improving lymphedema, and she was put on a high-protein diet.

\section{Discussion}

Pemphigoid is an autoimmune disease characterized by bullae formation resulting from the antigen-antibody reaction when autoantibodies bind to the hemidesmosome complex of the skin in the basement membrane zone.

The exact cause of pemphigoid is unknown, but it may be induced by certain drugs, including sulfasalazine, furosemide, and penicillin, and rarely by local burns, ultraviolet photo-

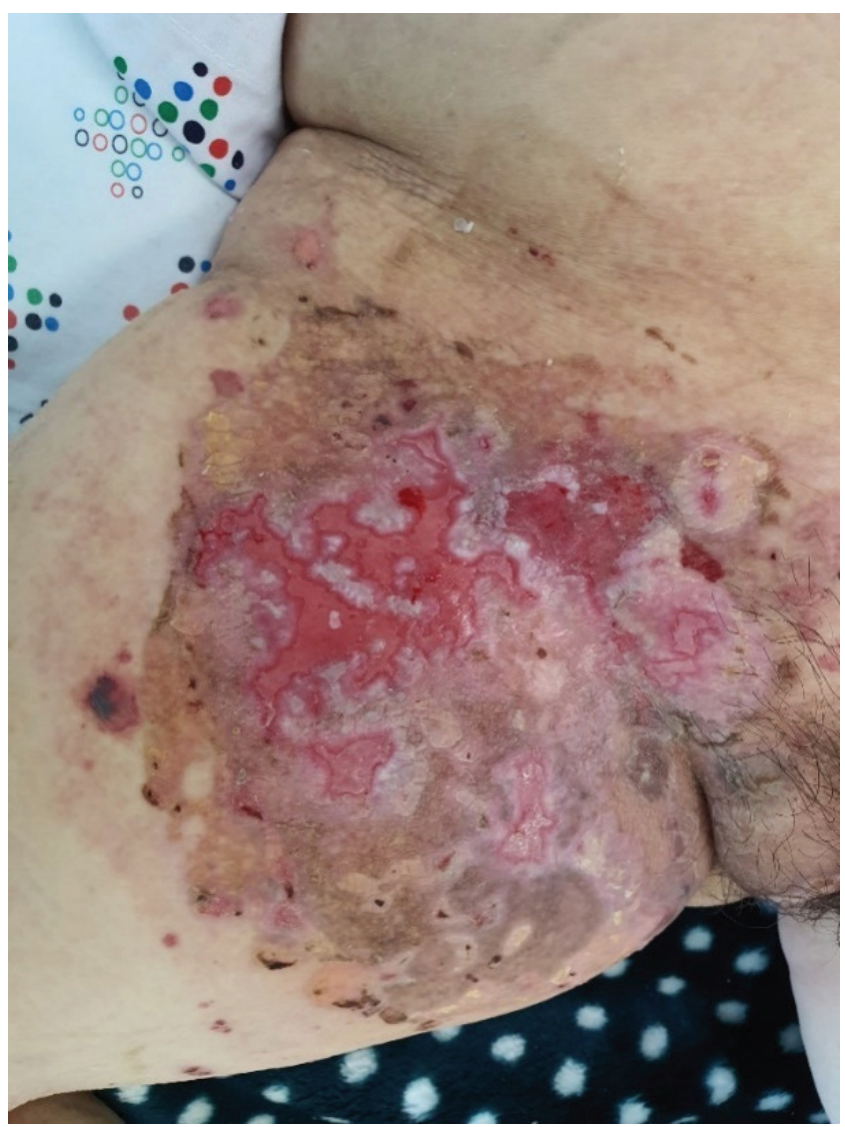

Fig. 1. On hospital day 4, diffuse and erythematous bullae size worsened in the right thigh. We only applied acyclovir cream on the lesion. therapy, and radiotherapy [1].

There are only a few reports of radiation-induced pemphigoid. It is a very rare complication in which radiation interferes with the sulfhydryl group in cells and changes the antigenic properties of the cell surface, leading to the antigenantibody reaction underlying pemphigoid.

The clinical signs of pemphigoid include bullae and rash, with or without itching. The outbreak occurs usually within 1 year after radiation treatment [2]. Pemphigoid cannot be diagnosed with clinical symptoms alone, and differential diagnoses including drug eruption, erythema multiforme, and acute viral infections such as varicella-zoster must be excluded using viral marker study, biopsy, and culture [3]. After radiation therapy, $95 \%$ of patients experience skin changes resulting from radiodermatitis (acute/chronic), erythema multiforme, Stevens-Johnson syndrome, and autoimmune diseases. Radiodermatitis is a common condition that occurs within 20 days after radiation, and clinical symptoms such as erythema spreading around the follicles and wrinkles at the site of radiation are commonly observed. Radiation-induced pemphigoid, however, is characterized by bullae formation at the irradiated site, beyond which the bullae can spread [4].

Such skin diseases can be worsened by several factors such as radiation dose, presence of other skin diseases, presence of a lesion due to previous chemical damage, obesity, smoking, and malnutrition. Patients receiving radiotherapy must be cautious of the above risk factors.

Treatment of radiation-induced pemphigoid using systemic

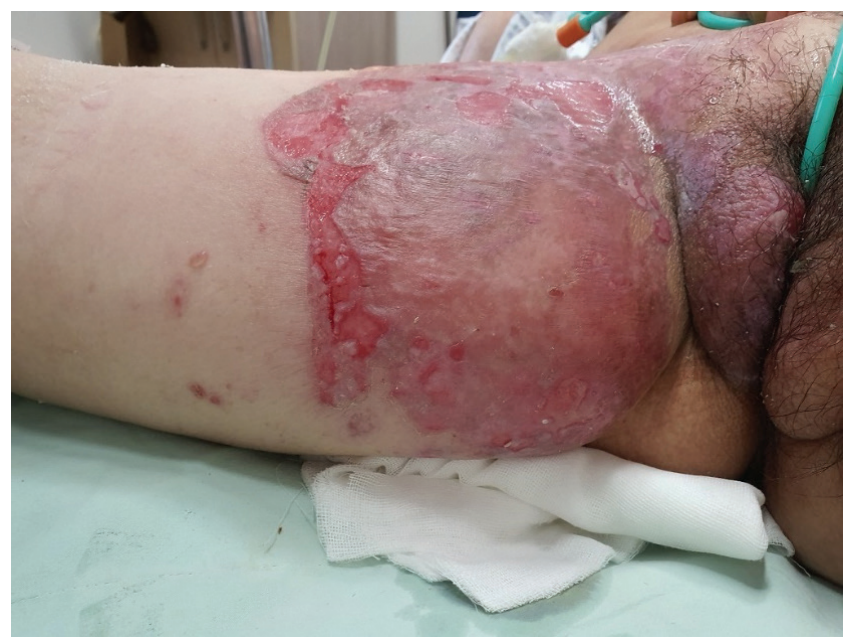

Fig. 2. After administering prednisolone and antibiotics, inflamed area healed and erythema did not spread. 


\section{Obstetrics \& Gynecology Science}

Vol. 63, No. 6, 2020

corticosteroids usually yields excellent results. Oral steroids at low daily doses of approximately $20-40 \mathrm{mg}$ are recommended, but continued use increases the risk of bone density decline and diabetes. Care must be taken during long-term use, and the dosage should be reduced once there is an improvement in the lesion [5]. Other treatment options include azathioprine, which is reported to be effective in maintaining relief of the lesion after steroid administration. Dapsone and sulfonamide can also be used, but without significant effect, and might be considered only for patients who cannot receive steroid treatment. Additionally, simultaneous antibiotic treatment may be helpful in the case of pemphigoid. It can prevent further infection caused by weakening of the skin barrier as pemphigoid progresses.

\section{Conflict of interest}

No potential conflict of interest relevant to this article was reported.

\section{Patient consent}

The patients provided written informed consent for the publication and the use of their images.

\section{References}

1. Shon W, Wada DA, Kalaaji AN. Radiation-induced pemphigus or pemphigoid disease in 3 patients with distinct underlying malignancies. Cutis 2016;97:219-22.

2. Isohashi F, Konishi K, Umegaki N, Tanei T, Koizumi M, Yoshioka Y. A case of bullous pemphigoid exacerbated by irradiation after breast conservative radiotherapy. Jpn J Clin Oncol 2011;41:811-3.

3. Mul VE, van Geest AJ, Pijls-Johannesma MC, Theys J, Verschueren TA, Jager JJ, et al. Radiation-induced bullous pemphigoid: a systematic review of an unusual radiation side effect. Radiother Oncol 2007;82:5-9.

4. Parikh SK, Ravi A, Kuo DY, Nori D. Bullous pemphigoid masquerading as acute radiation dermatitis: case report. Eur J Gynaecol Oncol 2001;22:322-4.

5. Nguyen T, Kwan JM, Ahmed AR. Relationship between radiation therapy and bullous pemphigoid. Dermatology 2014;229:88-96. 